\title{
HIGH-PRECISION ATTITUDE ESTIMATION METHOD OF STAR SENSORS AND GYRO BASED ON COMPLEMENTARY FILTER AND UNSCENTED KALMAN FILTER
}

\author{
Chengcheng Guo ${ }^{a}$, Xiaohua Tong ${ }^{a}{ }^{a,}$, Shijie Liu ${ }^{a}$, Sicong Liu ${ }^{a}$, Xiaojun Lu ${ }^{b}$, Peng Chen ${ }^{a}$, Yanming Jin ${ }^{a}$, Huan Xie ${ }^{a}$ \\ ${ }^{a}$ College of Surveying and Geo-Informatics, Tongji University, 1239 Siping Road, Shanghai, China - \\ xhtong@ tongji.edu.cn \\ ${ }^{\mathrm{b}}$ China International Engineering Consulting Corporation, Beijing, China
}

Commission III, ICWG III/II

KEY WORDS: Attitude Accuracy, Star Tracker, Gyro, Unscented Kalman Filter, Complementary Filter

\begin{abstract}
:
Determining the attitude of satellite at the time of imaging then establishing the mathematical relationship between image points and ground points is essential in high-resolution remote sensing image mapping. Star tracker is insensitive to the high frequency attitude variation due to the measure noise and satellite jitter, but the low frequency attitude motion can be determined with high accuracy. Gyro, as a short-term reference to the satellite's attitude, is sensitive to high frequency attitude change, but due to the existence of gyro drift and integral error, the attitude determination error increases with time. Based on the opposite noise frequency characteristics of two kinds of attitude sensors, this paper proposes an on-orbit attitude estimation method of star sensors and gyro based on Complementary Filter (CF) and Unscented Kalman Filter (UKF). In this study, the principle and implementation of the proposed method are described. First, gyro attitude quaternions are acquired based on the attitude kinematics equation. An attitude information fusion method is then introduced, which applies high-pass filtering and low-pass filtering to the gyro and star tracker, respectively. Second, the attitude fusion data based on CF are introduced as the observed values of UKF system in the process of measurement updating. The accuracy and effectiveness of the method are validated based on the simulated sensors attitude data. The obtained results indicate that the proposed method can suppress the gyro drift and measure noise of attitude sensors, improving the accuracy of the attitude determination significantly, comparing with the simulated on-orbit attitude and the attitude estimation results of the UKF defined by the same simulation parameters.
\end{abstract}

\section{INTRODUCITON}

The precise attitude estimation of high resolution remote sensing satellites is a key factor of high-precision geometric positioning and processing of high resolution satellite images. Nowadays, the discussion has revolved around attitude determination and control system (ADCS) because it is one of the most important subsystems of a satellite, since the accuracy of its mission depends on this subsystem capability. In other words, this subsystem represents the satellite visual sense and feeling in space (Mohammed et al., 2016). The objective of high-precision attitude determination is to calculate the space orientation of the satellite body or on-board payload coordinate system based on optimal attitude estimation algorithm, using measurement data obtained by sensors installed on the satellite body such as star sensor, sun sensor, infrared horizon sensor, gyroscope and the magnetometer, etc. In order to realize the precise estimation of satellite attitude, star sensor and gyroscope are used as main part of attitude determination system (Bae et al., 1998; Zhang et al., 2003). For some on-orbit high resolution remote sensing satellites, such as Landsat-7, GLAS and ALOS, the raw attitude data of star trackers and gyros are recorded and downlinked to the ground station for posteriori processing and batch filtering, improving the geometric positioning accuracy of remote sensing image (Iwata et al., 2005).

There are many algorithms for determining the attitude of a satellite, including attitude determination algorithms, such as TRIAD, Q-Method, Quest, Least Square, etc., attitude estimation algorithms, such as Kalman Filter, H-infinity Filter, Particle Filter, etc., and nonlinear observers, such as Sliding
Mode Observer, Luenberger observer, Backstepping observer, etc. Attitude determination algorithm obtains satellite attitude information based on observation vectors of star sensor. The attitude determination method does not require the priori information of the satellite attitude sensors. Results of attitude calculation have definite physical and geometric significance. However, it is difficult to overcome the uncertainty of reference vectors, such as measurement error of attitude sensors, installation error, bias error in general (Markley et al., 1978, 1993; Mortari et al., 1998). Establishing attitude estimation model including these uncertainties and processing weighted attitude observations of attitude sensors with different accuracies are almost impossible to implement. Since it was introduced by Kalman, the Kalman Filter has become a standard design tool for state estimation. Its popularity comes from its flexible approach that can generate good solutions to a wide range of estimation problems. For linear problems with white gaussian noise sources, it generates an optimal solution (Tang et al., 2014). The Kalman Filter theory greatly promoted the development of navigation technology combined with star tracker and gyro capabilities. The attitude estimation method based on Kalman Filter obtains the optimal state parameters by establishing the state equation and measurement equation of filter system. Extended Kalman Filter (EKF) was proposed by Anderson and Moore, becoming the most widely used nonlinear recursive filtering method in the field of attitude determination. However, the nonlinear state equation and the measurement equation of the EKF method may lead to biased state estimation or even filtering divergence because of local linearization approximation in the vicinity of the state prediction (Xiong et

\footnotetext{
${ }^{*}$ Corresponding author
} 
al., 2011; Markley et al., 2003; Crassidis et al., 2012). Aiming at the limitation of EKF method, the Unscented Kalman Filter applies Unscented Transformation (UT) to estimate the mean and covariance of the state, using a set of deterministic discrete sampling points (Sigma points) to approximate the distribution of the state variables (Van et al., 2001; Julier et al., 2002).The estimation accuracy of UKF is better than EKF when the system has nonlinear characteristics. Same as EKF, UKF is easily affected by model error and measurement noise, may diverge in the process of attitude filtering.

Complementary filter is a kind of attitude fusion algorithm for aircraft combined with integrating gyro and accelerometer. It is simple in calculation and can suppress gyro drift and track the attitude change (Euston et al., 2008; Cao et al., 2009). Because of the difficulty when determining the cut-off frequency of high-pass filtering and low-pass filtering, the accuracy of attitude determination is poor.

This study focuses on the filtering fusion of satellite attitude based on different attitude measurements of sensors. We proposed a novel attitude estimation method combined with star trackers and gyros based on Complementary Filter and Unscented Kalman Filter (CF\&UKF). The accuracy of the postprocessed attitude was evaluated by comparing it with the simulated attitude measurements of sensors, simulated attitude true value as well as attitude estimation results of UKF, respectively.

\section{METHODOLOGY}

As short-term attitude reference of satellite, gyroscope can continuously provide three axis angular velocity information of satellite body. However, due to the gyro drift, the uncertainty of initial conditions and the integral error, satellite attitude information contains error which increases with time. The star sensor can be used as a long-term reference for the satellite attitude, providing attitude quaternion information of the star tracker at a certain sampling frequency. The high frequency gyro measurement information is corrected by the measured values of low frequency star sensors. An information fusion filter estimator is proposed based on measurements of attitude sensors. The sequential processing algorithm is used to estimate the satellite attitude error quaternion and the gyro drift error, realizing high accuracy attitude estimation.

\subsection{Attitude Description}

Satellite attitude description parameters are the spatial pointing parameters of the satellite in a specific reference coordinate system, i.e., elements of exterior orientation of rigorous imaging model of high resolution satellite. Main expressions of attitude include directional cosine, Euler angle and quaternion etc. The direction cosine is not intuitive and lacks geometric meaning (Tu., 2003). The Euler angles of the rigid body rotation are used to represent the satellite attitude. However, singularities exist when rotation angle roll is equal to 90 degrees. In addition, trigonometric operations of Euler angles increase the complexity of attitude computation. Attitude quaternion is used as attitude parameters generally in space mission. The definition of the quaternion is shown in equation 1 :

$$
q=\left[\begin{array}{l}
q_{0} \\
q
\end{array}\right]=\left[\begin{array}{l}
q_{0} \\
q_{1} \\
q_{2} \\
q_{3}
\end{array}\right]=\left[\begin{array}{c}
\cos \frac{\varphi}{2} \\
e_{x} \sin \frac{\varphi}{2} \\
e_{y} \sin \frac{\varphi}{2} \\
e_{z} \sin \frac{\varphi}{2}
\end{array}\right]
$$

Where $\varphi$ is rotation angle, e is the rotation axis. Since quaternion has four components, which are not independent of each other. The unit constraint of quaternion should be imposed The conversion of the quaternion to the pitch angle $\theta$, roll angle $\varphi$, yaw angle $\kappa$ is shown in equation 2 :

$$
\left\{\begin{array}{l}
\theta=\arctan \left(\frac{2\left(q_{0} q_{2}-q_{1} q_{3}\right)}{q_{0}^{2}+q_{3}^{2}-q_{1}^{2}-q_{2}^{2}}\right) \\
\varphi=\arcsin \left[2\left(\mathrm{q}_{2} \mathrm{q}_{3}+\mathrm{q}_{0} \mathrm{q}_{1}\right)\right] \\
\kappa=\arctan \left(\frac{2\left(q_{0} q_{3}-q_{1} q_{2}\right)}{q_{0}^{2}-q_{3}^{2}-q_{1}^{2}+q_{2}^{2}}\right)
\end{array}\right.
$$

\subsection{Measurement Model of Attitude Sensors}

A typical star sensor output would be quaternion that represents the rotation between the ECI frame and the star sensor frame. Star sensor is one of the most complex attitude determination sensors, which essentially uses the CMOS or CCD sensor array to image the sky, and then use image processing techniques to pick out certain stars. Every star sensor has an on-board star catalogue which is used to identify the orientation of the spacecraft in inertial space by comparing the visible star pattern to the on-board catalogue. The low frequency error of star sensor exists in accordance with the orbital period originating from the change of thermal environment. The low frequency error is related to time, its magnitude can reach several tens of seconds. The magnitude of star tracker's measurement random noise, i.e., high-frequency error is angular seconds. By introducing the gyro data, the filtering method is adopted to reduce the influence of measurement noise on attitude determination accuracy.

The gyro measurement is the component of the angular velocity of the satellite relative to the inertial coordinate system in the satellite coordinate system. The gyro constant drift is the constant deviation between the gyro output angular velocity and the true angular velocity. The correlation drift is described by first order random walk, i.e., the random walk process driven by gauss white noise. The statistical properties of gyro drift vary with time. In order to improve the accuracy of attitude estimation system, gyro drift must be estimated and corrected in real time.

\subsection{Attitude Kalman Filter In Quaternion Space}

The attitude kinematics equation describes the variation of attitude parameters in the attitude maneuver process. It can be described by the following formula: 


$$
\left[\begin{array}{c}
\dot{q_{0}} \\
\dot{q_{1}} \\
\dot{q_{2}} \\
\dot{q_{3}}
\end{array}\right]=\frac{1}{2} \Omega(w) q=\frac{1}{2}\left[\begin{array}{cccc}
0 & -w_{x} & -w_{y} & -w_{z} \\
w_{x} & 0 & w_{z} & -w_{y} \\
w_{y} & -w_{z} & 0 & w_{x} \\
w_{z} & w_{y} & -w_{x} & 0
\end{array}\right]\left[\begin{array}{l}
q_{0} \\
q_{1} \\
q_{2} \\
q_{3}
\end{array}\right]
$$

For the calculation of the attitude quaternion differential equation, in order to meet the requirements of precision and speed, the first-order Runge-Kutta method is used. The attitude parameters must be normalized to meet the characteristics of the unit quaternion after the updating of quaternion. The vector part of the quaternion and the gyro drift deviation are used as the estimate state, and filter state equation can be obtained.

$$
\left[\begin{array}{c}
\dot{Q_{e}} \\
\dot{\Delta b}
\end{array}\right]=\left[\begin{array}{cc}
-[\omega \times] & -\frac{1}{2} I_{3} \\
0_{3 \times 3} & 0_{3 \times 3}
\end{array}\right]\left[\begin{array}{c}
Q_{e} \\
\Delta b
\end{array}\right]+\left[\begin{array}{cc}
-\frac{1}{2} I_{3} & 0_{3 \times 3} \\
0_{3 \times 3} & I_{3}
\end{array}\right]\left[\begin{array}{l}
v \\
n
\end{array}\right] \text { (4) }
$$

Where $\dot{Q}_{e}$ is the vector part of the error quaternion, $\Delta \dot{b}$ is the difference between true value and estimate value of gyro drift, $[w \times]$ is the cross product matrix of gyro angular velocity. The above formula is the state transfer equation. The indirect form (error state) is applied in this paper, reducing the attitude estimate to three independent variables. The singularity of covariance matrix in the filtering process caused by nonindependence of quaternion variables is avoided.

The measurement equation of EKF is established, which is to establish the relation between the filter state and the system measurement value.

$$
\left\{\begin{array}{c}
\Delta q_{\text {out }}=\Delta q+n_{\text {out }}=\left[\begin{array}{ll}
I & 0
\end{array}\right]\left[\begin{array}{l}
\Delta q \\
\Delta b
\end{array}\right]+n_{\text {out }} \\
\Delta Z=H X+V
\end{array}\right.
$$

Where $\Delta Z$ is the difference between quaternion measurement of star sensor and the estimated attitude parameters.

\subsection{Attitude Estimation Method Based On Complementary Filter and Unscented Kalman Filter}

Star tracker is insensitive to the high frequency attitude variation due to the measure noise and satellite jitter, but the low frequency attitude motion can be determined with high accuracy. Gyro, as a short-term reference to the satellite's attitude, is sensitive to high frequency attitude change, but due to the existence of gyro drift and integral error, the attitude determination error increases with time. In the field of multisensors fusion, Complementary Filtering is an alternative to Kalman Filtering. Complementary filter is simpler which does not interfere with the original measurement signal and effectively remove the measurement noise of the sensor at the same time. Complementary filter system is an open loop structure rather than a feedback structure, which made the phenomenon of filtering divergence will never happen. However, the complementary filter works only when noise frequency characteristics are complementary. The attitude characteristics of different attitude sensors are fused by applying high-pass filtering and low-pass filtering to the gyro and star tracker, respectively. The initial fusion attitude value of the star sensor and gyro based on complementary filtering compensates the gyro drift and suppress the filtering divergence, improving the sensitivity of star sensor to high-frequency attitude change. In practical applications, the cut-off frequency of the filter needs to be determined according to the noise level of sensors, i.e. the weights of sensors attitude fusion. The process of attitude data initial fusion based on complementary filtering is shown in figure 1 .

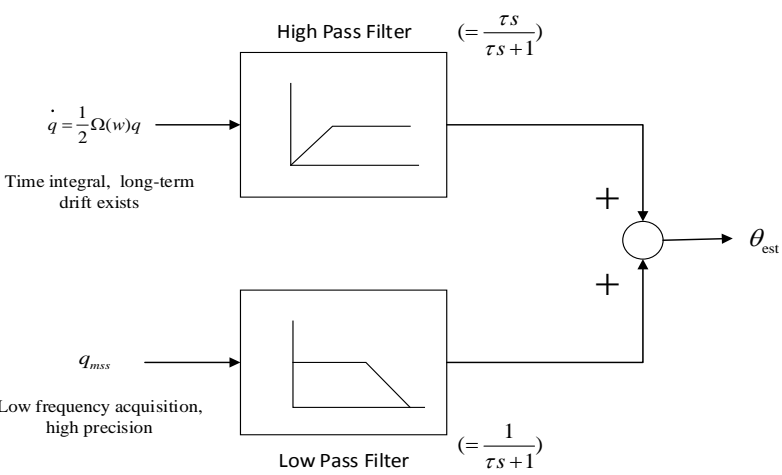

Figure 1. Sketch of attitude initial fusion based on complementary filtering

The attitude fusion data based on $\mathrm{CF}$ are introduced as the observed values of UKF system in the process of measurement updating, which can improve the accuracy of system measurements and accelerate the rate of convergence. The fusion attitude based on CF can suppress gyro drift and attitude sensors measurement noise. Due to high measurement frequency of inertial gyro, Firstly, the attitude data is updated based on the kinematic equation by using the gyro angular velocity data. Secondly, when the star sensor attitude data is acquired, filtering system measurement is constructed based on complementary filter. Thirdly, the UKF process is implemented by using constructed system measurements. Finally, the estimated error quaternion and gyro drift increment are obtained which will be used in next loop. It is important to note that at the beginning of each filter cycle, the disturbance state (error state) need to be reset to 0 . The sketch of filter method is shown in figure 2.

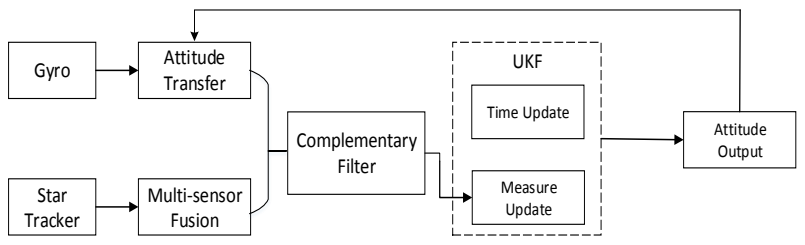

Figure 2. Flow chart of attitude estimation based on CF\&UKF

The mean and variance of the nonlinear system is estimated by means of unscented transformation in UKF (Crassidis et al., 2003). In the filtering process, $2 \mathrm{n}+1$ sampling points around 
state variables (Sigma points) and corresponding weights need to be calculated first. The mean value and variance of state and measurements by one step predicting can be calculated based on sigma points which represent the filtering state. Namely, the unscented transformation (UT) is applied to the state equation and the measurement equation, respectively. The time updating procedure and measurement updating procedure is the same as EKF. In the process of filtering, the covariance matrix of system noise is the state prediction error caused by the deviation between state equation and the real system model, the covariance matrix of measurement noise is the error caused by the deviation of measurement model and the real output, which both determine the robustness of the filter and the accuracy of the state estimation.

\section{EXPERIMENT AND ANALYSIS}

\subsection{Simulation Experiment of Attitude Estimation}

In order to verify the accuracy and effectiveness of the proposed algorithm, the attitude simulation experiments are carried out. The system simulation step is $0.1 \mathrm{~s}$, total simulation time is 180 second, dynamic angular velocity $\omega_{b i}=\left[\begin{array}{lll}1 & 0 & 0\end{array}\right]^{T} \mathrm{deg} / \mathrm{s}$, attitude initial value $q=\left[\begin{array}{llll}1 & 0 & 0 & 0\end{array}\right]^{T}$, gyro constant drift is $1 \mathrm{deg} / \mathrm{h}$, gyro measurement noise mean square error is $1 \mathrm{deg} / \mathrm{h}$, mean square error of gyro random drift is $0.1 \mathrm{deg} / \mathrm{h}$, the measurement frequency of gyro is $10 \mathrm{~Hz}$. The measurement error of star sensor quaternion is gauss noise, whose standard deviation is 1e-5, equivalent to 4.1 seconds attitude angle measurement error. The measurement frequency of star tracker is $4 \mathrm{~Hz}$. The initial state vector is zero including error quaternion and correction of gyro drift. Initial state error covariance matrix $\mathrm{P}=1 \mathrm{e}-6 I_{6 * 6}$. Based on the proposed method, the quaternion q 3 of gyro, star tracker, filtering method and true attitude are shown in figure 3.

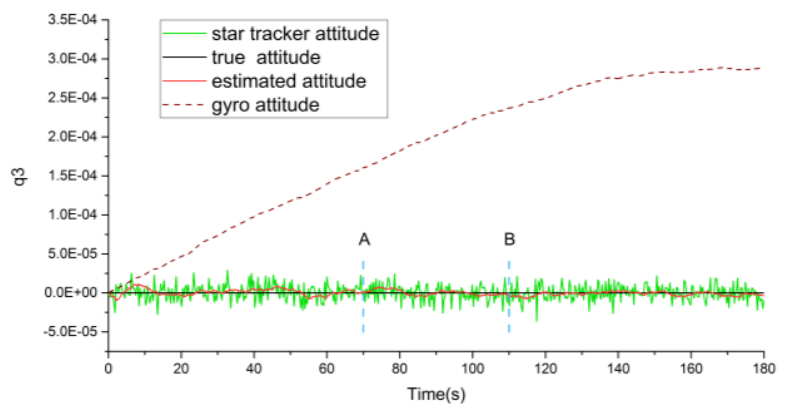

Figure 3. The accuracy comparison of quaternion q3 by different attitude estimation methods.

In the process of recursive filtering, gyro angular velocity is corrected by using the obtained estimated state, improving the accuracy of gyro measurements. The attitude estimation algorithm based on the proposed method is effective to estimate the gyro constant drift which is about 4e-6rad/s. The result is shown in figure 4 . The standard deviation of roll, pitch and yaw is $1.2266,1.3064$ and 1.3080 , respectively.

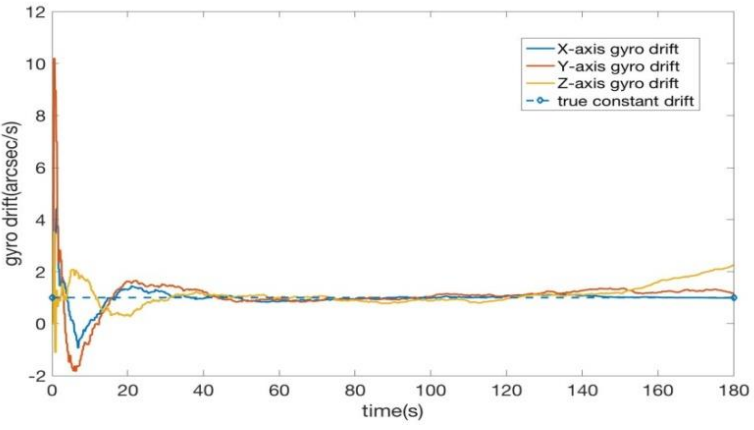

Figure 4. The estimation of gyro drift based on the proposed method

\subsection{Analysis of Attitude Simulation Results}

The results of attitude estimation accuracy (RMSE) by using same simulation parameters based on different methods are shown in the following table.

\begin{tabular}{llll}
\hline $\begin{array}{l}\text { Estimation } \\
\text { method }\end{array}$ & Roll & Pitch & Yaw \\
\hline Star tracker & arc sec & arc sec & arc sec \\
Gyro & 102.7798 & 4.1260 & 4.1300 \\
EKF & 1.5588 & 1.6817 & 103.6970 \\
UKF & 1.5418 & 1.6503 & 1.6435 \\
CF\&UKF & 1.2266 & 1.3064 & 1.3080 \\
\hline
\end{tabular}

Table 1. Statistics of attitude estimation accuracy

Based on the same simulation parameters, the attitude estimation error of UKF is calculated, in which the standard deviation of roll is 1.5418 seconds, the standard deviation of pitch is 1.6503 seconds, and the standard deviation of yaw is 1.6435 seconds. The attitude measurement error of star tracker in the form of Eular angle is about 4.1 arcsec. Due to the presence of constant drift and correlation drift, the measurement error of gyro attitude angles are 102.7788 seconds, 48.9967 seconds, 103.6970 seconds, respectively. In the proposed method, the filter divergence is suppressed due to the introduction of complementary filter substructure in open-loop form into the UKF structure. In the process of iterative filtering, when attitude measurements of star sensor are introduced, they are fused with the attitude filtering values obtained from the previous step. Thus, the attitude measurement value of the current state is obtained, then the attitude UKF process is executed. In the process of initial attitude fusion, the weight determination is a key factor to the accuracy of attitude estimation. Therefore, the high and low pass filter in the complementary system should have a proper cut-off rate to obtain stable and high-accuracy attitude measurements. In the simulation experiment, the cut-off frequency is $2 \mathrm{HZ}$ based on the experience and tests. Through simulation experiments, it's found that the accuracy of proposed method is affected by the updating frequency of star sensor especially. For example, the accuracy of roll angle is more than 4 arc seconds and less than 1 arc second when the measurement frequency of star tracker is 1 $\mathrm{Hz}$ and $10 \mathrm{~Hz}$, respectively. It is significant to improve the measurement frequency of star sensor for the improvement of attitude estimation accuracy. 


\section{CONCLUSION AND FUTURE WORK}

In this paper, a new attitude estimation method for star sensor and gyro based on Complementary Filter and Unscented Kalman Filter is proposed to solve the problem that the attitude estimation based on Kalman Filter is easy to diverge and the convergence speed is slow. By introducing the attitude fusion value based on Complementary Filter into the measurement updating process of Unscented Kalman Filter, the attitude estimation accuracy is improved. It can be seen from the simulation results that the proposed method can effectively suppress the gyro drift and the sensor measurement noise, fusing the preponderant characteristics of two attitude sensors. In the process of attitude maneuver, the gyro drift is estimated effectively, verifying the correctness of the algorithm. It is necessary to point out that the determination of the cut-off frequency of complementary filtering is still a problem that needs to be solved. At the same time, the accuracy of proposed method is largely affected by the updating frequency of star sensor. In the future work, the method will be applied to the processing of actual satellite attitude data, in order to improve the accuracy of remote sensing satellite geometric positioning.

\section{ACKNOWLEDGEMENTS}

This paper was substantially supported by the National Natural Science Foundation of China (Project nos. 41631178, 41325005, 41401531 and 41601414), and the Fundamental Research Funds for the Central Universities.

\section{REFERENCES}

Mohammed, M., Bellar, A., Adnane, A., Boussadia, H., 2016. Performance analysis of attitude determination and estimation algorithms applied to low earth orbit satellites. Ukacc, International Conference on Control (pp.1-6). IEEE.

Bae, S., 1998. Glas Spacecraft Attitude Determination Using CCD Star Tracker and 3-AXIS Gyros.

Zhang, Y., Wang, H., and Chen, J., 2003. FOG/Star-sensor Combination Technology for Satellite Three-axis Attitude Determination. Journal of Chinese Inertial Technology. 11(2):1-4

Markley, F. L., Lerner, G.M., 1978. Three-axis attitude determination methods. Astrophysics and Space Science Library. Astrophysics and Space Science Library.

Mortari, D., 1998. Euler-q Algorithm for Attitude Determination from Vector Observations. Journal of Guidance Control \& Dynamics, 21(2):328-334.

Markley, F. L., 1993. Attitude Determination from Vector Observations: A Fast Optimal Matrix Algorithm. Journal of the Astronautical Sciences, 41(2):261-280.

Xiong, K., Liang, T., Lei, Y., 2011. Multiple model Kalman filter for attitude determination of precision pointing spacecraft. Acta Astronautica, 68(7):843-852.

Markley, F. L., 2003. Attitude Error Representations for Kalman Filtering. Journal of Guidance Control \& Dynamics, 26(2):311-317.
Crassidis, J. L., Markley, F. L., Cheng, Y., 2012. Survey of Nonlinear Attitude Estimation Methods. Journal of Guidance Control \& Dynamics, 30(1):12-28.

Crassidis, J. L., Markley, F. L., 2003. Unscented Filtering for Spacecraft Attitude Estimation. AIAA Guidance, Navigation, and Control Conference and Exhibit. 536-542.

Van, D. M. R., Wan, E. A., 2001. The square-root unscented Kalman filter for state and parameter-estimation. IEEE International Conference on Acoustics, Speech, and Signal Processing, Proceedings. IEEE, 3461-3464 vol.6.

Julier, S. J., 2002. The scaled unscented transformation. American Control Conference, Proceedings of the. IEEE, 45554559 vol.6.

Euston, M., Coote, P., Mahony, R., 2008. A complementary filter for attitude estimation of a fixed-wing UAV. International Conference on Intelligent Robots and Systems. IEEE, 340-345.

Cao, D., Qu, Q., Li, C., 2009. Research of Attitude Estimation of UAV Based on Information Fusion of Complementary Filter. International Conference on Computer Sciences and Convergence Information Technology. IEEE Xplore, 12901293.

Tang, X., Xie, J., Wang, X., 2014. High-Precision Attitude Post-Processing and Initial Verification for the ZY-3 Satellite. Remote Sensing, 7(1):111-134.

Iwata, T., 2005. Precision attitude and position determination for the Advanced Land Observing Satellite (ALOS). Proceedings of SPIE - The International Society for Optical Engineering, 5659(6):1-20.

Tu, S., 2003. Satellite Attitude Dynamics and Control. China Astronautic Publishing House. 